\title{
Erratum
}

\section{Structural and magnetic studies on mono- and polynuclear chromium ascorbate complexes}

\author{
Birgül Zümreoglu-Karan, Ahmet N. Ay and Canan Ünaleroglu \\ Department of Chemistry, Hacettepe University, Beytepe Campus, 06800 Ankara, Turkey
}

Tezer Firat

Department of Physics Engineering, Hacettepe University, Beytepe Campus, 06800 Ankara, Turkey

Thomas Ristau and Wolfgang Jabs

Institut für Chemie u. Biochemie, E. M. A. Universität Greifswald, D-17489 Greifswald, Germany

\section{Transition Metal Chemistry (2005) 30:451-459}

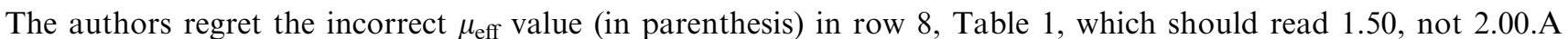
central section in the manuscript describes the magnetic behaviour of mono- and polynuclear chromium ascorbate complexes. The authors would like to revise the third paragraph in the second column, page 454:

Original Paragraph "The molecular magnetic susceptibility of the mononuclear complex (1) seems not to obey the Curie-Weiss law. The slow increase (especially at low temperatures) of the effective molecular magnetic moment with temperature from $1.8 \mu_{\mathrm{B}}$ at $T=2 \mathrm{~K}$ up to $3.51 \mu_{\mathrm{B}}$ at $T=300 \mathrm{~K}$ (Figure 3a) cannot be explained even with very large zero field splitting values $\left(D>100 \mathrm{~cm}^{-1}\right)$. The smaller value of the effective magnetic moment at $T=$ $300 \mathrm{~K} \mu_{\mathrm{eff}}=3.51 \mu_{\mathrm{B}}$, as the expected spin only value $\mu_{\text {s.o. }}=3.87 \mu_{\mathrm{B}}$, can be due to some spin orbital interaction and impurity contribution (a $S=1 / 2$ signal was observed in the EPR measurement). Simulations for a $\mathrm{Cr}$ (III) with $\mathrm{d}^{3}$ configuration in axially distorted surroundings [44] have similarly showed a slow increase of $\mu_{\text {eff }}$ at low temperatures but no proper fitting was achieved in this case. Other possible explanations for this unusual behaviour can be anisotropy or intermolecular interactions which may also help explaining the difficulty to fit the magnetic measurements of (2) (Figure 3b) and (3) (Figure $3 \mathrm{c})$. The results indicate a small antiparallel magnetic interaction between the $\mathrm{Cr}(\mathrm{III})$ centers $\left(\mathrm{d}^{3}, S=3 / 2\right)$ with an interaction coupling $J=11.5 \mathrm{~cm}^{-1}$ for (2) and $J=6.7 \mathrm{~cm}^{-1}$ for (3). The results are summarized in Table 4."
Revised Paragraph "The molecular magnetic susceptibility of the mononuclear complex (1) seems not to obey the Curie-Weiss law. The effective molecular magnetic moment slowly increased with temperature (especially at low temperatures) from $1.8 \mu_{\mathrm{B}}$ at $T=2 \mathrm{~K}$ up to $3.51 \mu_{\mathrm{B}}$ at $T=300 \mathrm{~K}$ (Figure 3a). Simulations for a $\mathrm{Cr}$ (III) with $\mathrm{d}^{3}$ configuration in axially distorted surroundings [44] have similarly showed a slow increase of $\mu_{\mathrm{eff}}$ at low temperatures but no proper fitting was achieved in this case. The smaller value of the effective magnetic moment at $T=300 \mathrm{~K} \mu_{\text {eff }}=3.51 \mu_{\mathrm{B}}$, as the expected spin only value $\mu_{\text {s.o. }}=3.87 \mu_{\mathrm{B}}$, can be due to some spin orbital interaction and impurity contribution (a $S=1 / 2$ signal was observed in the EPR measurement). Since the compounds were obtained from aqueous solutions by vacuum drying or by precipitation with the addition of cold acetone, impurity contribution is more likely. Other possible explanations for this unusual temperature dependence of magnetic susceptibility can be anisotropy or intermolecular interactions which may also help explaining the difficulty to fit the magnetic measurements of (2) (Figure 3b) and (3) (Figure 3c). The results indicate a small antiparallel magnetic interaction between the $\mathrm{Cr}(\mathrm{III})$ centers $\left(\mathrm{d}^{3}, S=3 / 2\right)$ with an interaction coupling $\mathbf{J}=11.5 \mathrm{~cm}^{-1}$ for (2) and $J=6.7 \mathrm{~cm}^{-1}$ for (3). The results are summarized in Table 4." 